\title{
An Efficient Approach for the Synthesis of Pyrazolo [1,2,a] $[1,2,4]$ Triazole-1,3-Diones Using an Electrochemical Cell
}

\author{
KHATEREH KHANDAN-BARANI ${ }^{*}$, MOHAMMAD DODANGEH ${ }^{2}$, \\ MEHRNOOSH KANGANI ${ }^{2}$ and MALEK-TAHER MAGHSOODLOU ${ }^{2 *}$ \\ 'Department of Chemistry, Islamic Azad University, Zahedan Branch, \\ PO Box 98135-978, Zahedan, Iran. \\ ${ }^{2}$ Department of Chemistry, Faculty of Science, University of Sistan and Baluchistan, \\ P. O. Box: 98135-674, Zahedan, Iran.
}

http://dx.doi.org/10.13005/ojc/320252

(Received: December 08, 2015; Accepted: January 15, 2016)

\begin{abstract}
A mild and efficient method was applied for the one-pot three-component synthesis of pyrazolo[1,2-a][1,2,4]triazole-1,3-diones from the condensation between arylaldehydes, malononitrile and 4-phenylurazole using electrolysis in an undivided cell in the presence of sodium bromide as an electrolyte. This procedure has many advantages such as : no need to catalyst, clean work-up, short reaction time and high yield. The products were obtained just with the simple filtration.
\end{abstract}

Keywords: Pyrazolo[1,2-a][1,2,4]triazole-1,3-dione; 4-phenylurazole; Malononitrile; Electrolysis.

\section{INTRODUCTION}

Many of heterocyclic compounds containing nitrogen have significant synthetic and biological importance, which act as polyfunctionalized fragments, agrochemicals, and pharmaceuticals ${ }^{1-10}$. Pyrazolourazoles as their fused derivatives with diverse structures have attracted much interest because of their wide range of biological properties such as analgesic, antibacterial, anti-inflammatory, antidiabetic, and psychoanaleptic activities ${ }^{11-16}$ (Figure 1). Many methods have been reported for their synthesis in recent years ${ }^{17-26}$, and development of new approaches for their synthesis seems to be interesting challenge.

For preparing biologically active compounds in organic, combinatorial, and medicinal chemistry the electrosynthetically multicomponent reactions (EMCRs) have been used widely ${ }^{27}$. Due to the electron transfer between an electrode and the substrate molecules, the formation of highly reactive intermediates is achieved under mild conditions, avoiding reductive or oxidant agents as well as acids, bases and related waste by-products so that it can be one of the various fields in green chemistry ${ }^{28 .}$ 
In continue of our research on multi-component reactions ${ }^{29-32}$, Herein, we report an efficient and green synthesis of pyrazolo[1,2-a][1,2,4]triazole1,3-dione derivatives using an electrochemical cell (scheme 1).

\section{MATERIALS AND METHOD}

All reagents and solvents were obtained from Fluka and Merck and used without further purification. TLC was performed on Silica-gel Polygram SILG/UV 254 plates. Melting points and IR spectra were measured on an Electro thermal 9100 apparatus and a JASCO FT-IR-460 plus spectrometer. Controlled-current coulometer and preparative electrolysis were performed using a SAMA potentiostate/galvanostate (Zahedan, Iran), respectively. The ${ }^{1} \mathrm{H}$ NMR spectra were obtained on Bruker DRX-400 Advance instruments with DMSO.
General procedure for the synthesis of 7-amino-1,3-dioxo-2-phenyl-2,3-dihydro- $1 \mathrm{H}, 5 \mathrm{H}$ pyrazolo(1,2-a) $(1,2,4)$ triazole-6-carbonitrile

A mixture of arylaldehyde $(1 \mathrm{mmol})$, malononitrile $(1 \mathrm{mmol}), 4$-phenylurazole $(1 \mathrm{mmol})$ and $\mathrm{NaBr}(0.05 \mathrm{~g}, 0.5 \mathrm{mmol})$ in $\mathrm{EtOH}(20 \mathrm{~mL})$ was stirred with a magnetic stirrer and electrolyzed in an undivided cell equipped with a graphite anode, and an iron cathode at ambient temperature under a constant current density of $10 \mathrm{~mA} / \mathrm{cm}^{2}$ ( electrodes square $5 \mathrm{~cm}^{2}$ ), until the catalytic quantity of $0.1 \mathrm{~F} / \mathrm{mol}$ of electricity was passed. After electrolysis process, the mixture was filtered, then it was rinsed twice with cold ethanol to obtained corresponding product.

\section{Analytical data for the selected compounds}

7-amino-5-(3-nitrophenyl)-1,3-dioxo-2-phenyl-2,3dihydro- $1 \mathrm{H}, 5 \mathrm{H}$-pyrazolo $(1,2-\mathrm{a})(1,2,4)$ triazole-6-<smiles>O=C1C(c2ccccc2)C(=O)N2C3C(=CCN12)CCCC3O</smiles>

antimicrobial agent<smiles>CCCCCC1C(=O)N2C=C(c3ccccc3)c3ccccc3N2C1=O</smiles>

Cintazone anti-inflammatory agent<smiles>CC12C(=CCN3C(=O)C(c4ccccc4)C(=O)N31)C(=O)C(O)C2O</smiles>

Inhibitor of HSP induction

Fig. 1: pyrazolourazoles with diverse structures<smiles>[R]c1ccc(C(=O)NCCCC)cc1</smiles>

1<smiles>N#CC[C+]#N</smiles>

2<smiles>O=c1[nH][nH]c(=O)n1-c1ccccc1</smiles>

3

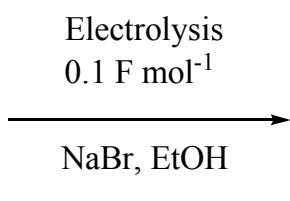<smiles>[R][R]1ccc(C2C(C#N)=C(N)n3c(=O)n(-c4ccccc4)c(=O)n32)cc1</smiles>

$4 a-1$

Scheme 1: Synthesis of pyrazolo $[1,2-a][1,2,4]$ triazole-1,3-dione derivatives with an electrochemical cell 
carbonitrile (4b)

White powder, m.p: $\left(>300^{\circ} \mathrm{C}\right)$; I IR $(\mathrm{KBr})\left(v_{\max } / \mathrm{cm}^{-1}\right)$ : 3430, 3314, 2189, 1766, 1715. 'H NMR (400 MHz, DMSO- $\left.d_{6}\right) \delta_{\mathrm{H}} 6.094(1 \mathrm{H}, \mathrm{s}, \mathrm{CH}), 7.47-8.37(11 \mathrm{H}, \mathrm{m}$, $\mathrm{H}-\mathrm{Ar}$ and $\mathrm{NH}_{2}$ ).

7-amino-5-(3-chlorophenyl)-1,3-dioxo-2-phenyl2,3-dihydro- $1 \mathrm{H}, 5 \mathrm{H}$-pyrazolo $(1,2-\mathrm{a})(1,2,4)$ triazole6-carbonitrile (4c)
White powder, m.p: $\left(>300^{\circ} \mathrm{C}\right)$; $\mathrm{IR}(\mathrm{KBr})\left(v_{\max } / \mathrm{cm}^{-1}\right)$ : 3450, 3338, 2200, 1778, 1715. ${ }^{1} \mathrm{H}$ NMR $(400 \mathrm{MHz}$, DMSO- $\left.d_{6}\right) \delta_{H} 6.09(1 \mathrm{H}, \mathrm{s}, \mathrm{CH}), 7.47-8.37(11 \mathrm{H}, \mathrm{m}$, $\mathrm{H}-\mathrm{Ar}$ and $\mathrm{NH}_{2}$ )

7-amino-5-(2-chlorophenyl)-1,3-dioxo-2-phenyl2,3-dihydro- $1 \mathrm{H}, 5 \mathrm{H}$-pyrazolo $(1,2-\mathrm{a})(1,2,4)$ triazole6-carbonitrile (4I)

White powder, m.p: $\left(>300^{\circ} \mathrm{C}\right)$.; IR $(\mathrm{KBr})\left(v_{\max } / \mathrm{cm}^{-1}\right)$ :

Table 1: Optimization of Reaction Conditions for the Synthesis of 7-Amino1,2,3,5-Tetrahydro-1,3-Dioxo-2,5-Diphenylpyrazolo[1,2-A][1,2,4]Triazole-6Carbonitrile

\begin{tabular}{ccccccc}
\hline Entry & I (mA) & $\begin{array}{c}\text { Current } \\
\text { density } \\
\left(\mathbf{m A} / \mathbf{c m}^{2}\right)\end{array}$ & $\begin{array}{c}\text { Time } \\
(\mathbf{m i n})\end{array}$ & $\begin{array}{c}\text { catalyst } \\
\text { passed } \\
(\text { F/mol) }\end{array}$ & $\begin{array}{c}\text { Electricity } \\
(\%)\end{array}$ & Yields \\
\hline 1 & 5 & 1 & 250 & - & 0.1 & 50 \\
2 & 10 & 2 & 160 & - & 0.1 & 65 \\
3 & 20 & 4 & 50 & - & 0.1 & 80 \\
4 & 50 & 10 & 33 & - & 0.1 & 93 \\
5 & 75 & 15 & 23 & - & 0.1 & 80 \\
\hline
\end{tabular}



Scheme 2: Proposed mechanism for the synthesis of pyrazolo[1,2-a][1,2,4]triazole-1,3-dione derivatives using electrolysis in the presence of sodium bromide as an electrolyte 
Table 2: Synthesis Of Pyrazolo[1,2-A][1,2,4]Triazole-1,3-Dione Derivatives

\begin{tabular}{|c|c|c|c|c|c|}
\hline Entry & Aldehyde & Product & Yield(\%) & $\mathrm{m} . \mathrm{p}\left({ }^{\circ} \mathrm{C}\right)$ & Reference [24] \\
\hline 1 & & $4 a$ & 93 & $>300$ & $>300$ \\
\hline 2 & & $4 b$ & 93 & $>300$ & $>300$ \\
\hline 3 & & $4 c$ & 85 & $>300$ & $>300$ \\
\hline 4 & & $4 d$ & 90 & $>300$ & $>300$ \\
\hline 5 & & $4 e$ & 85 & $>300$ & $>300$ \\
\hline 6 & & $4 f$ & 90 & $>300$ & $>300$ \\
\hline 7 & & $4 \mathrm{~g}$ & 90 & $>300$ & $>300$ \\
\hline 8 & & $4 \mathrm{~h}$ & 89 & $>300$ & $>300$ \\
\hline 9 & & $4 i$ & 83 & $>300$ & $>300$ \\
\hline 10 & & $4 j$ & 87 & $>300$ & $>300$ \\
\hline 11 & & $4 \mathrm{k}$ & 90 & $>300$ & $>300$ \\
\hline 12 & & $4 I$ & 93 & $>300$ & $>300$ \\
\hline
\end{tabular}


3400, 3319, 1778, 1725. 'H NMR (400 MHz, DMSO$\left.d_{6}\right) \delta_{\mathrm{H}} 6.27(1 \mathrm{H}, \mathrm{s}, \mathrm{CH}), 7.44-7.69(11 \mathrm{H}, \mathrm{m}, \mathrm{H}-\mathrm{Ar}$ and $\mathrm{NH}_{2}$ ).

\section{RESULTS AND DISCUSSION}

To optimized the reaction conditions, the condensation between benzaldehyde, malononitrile and 4-phenylurazole was chosen as model reaction. The reactive mixture was stirred at room temperature, and this progress was monitored by TLC. The reaction is performed in alcoholic solvents in the presence of sodium bromide as an electrolyte. Various current quantities were applied under the mentioned conditions. As can be seen in Table 1, excellent conversions of the starting materials were obtained under $10 \mathrm{~mA} / \mathrm{cm} 2$ current densities after $0.1 \mathrm{~F} / \mathrm{mol}$ of electricity had passed. The current density of $10 \mathrm{~mA} / \mathrm{cm} 2, \mathrm{I}=50 \mathrm{~mA}$, electrode surface Scheme 1.

Using mentioned optimized reaction, the reaction were explored for the synthesis of a wide variety of pyrazolo[1,2-a][1,2,4]triazole-1,3-diones using aromatic aldehydes, malononitriles and 4-phenylurazol. The results are summarized in Table 2. As shown in Table 2, the products were obtained in excellent yields.

We proposed mechanism for the preparation of pyrazolo[1,2-a][1,2,4]triazole-1,3dione derivatives. First, deprotonation of an alcohol at the cathode leads to the formation of the alkoxide anion $^{33}$. It's subsequent reaction in solution with malononitrile gives rise to the malononitrile anion. Then, Knoevenagel condensation of aldehyde 1, with the malononitrile anion takes place in the solution with the elimination of water and the formation of the corresponding a-cyanocinnamo-nitrile derivatives A. The subsequent hydroxide-promoted Michael addition of 4-phenylurazole 3 to the electron-deficient Knoevenagel adduct $\mathbf{A}$ followed by intramolecular cyclization results in the corresponding products 4 , with regeneration of the alkoxide anion as the last step, which continues the catalytic chain process by the interaction with the next molecule of malononitrile.

\section{CONCLUSION}

The pyrazolo[1,2-a][1,2,4]triazole-1,3-dione derivatives were synthesised in the presence of sodium bromide as an electrolyte under neutral and mild conditions. The main advantages of this method are the very short reaction time, high yields, simple work-up, use of non- hazardous organic solvent and catalyst.

\section{ACKNOWLEDGMENT}

We gratefully acknowledge financial support from the Research Council of the University of Sistan and Baluchestan.

\section{REFRENCES}

1. Franklin, E. C. Heterocyclic Chem Rev. 1935, 16, 305-361.

2. Bergstrom,F. W. Chem Rev 1944, 35, 77277.

3. Lichtenthaler, F. W. Acc Chem Res. 2002, 35, 728-737.

4. Dömling, A.; Ugi, I.; Angew Chem Int Ed. 2000,39, 3168-3210.

5. Dömling, A. Chem Rev. 2006, 106, 17-89.

6. Sheibani,H.; Babaie, M. Synth Commun. 2010, 40, 257.

7. Sheibani, H.; Seifi, M.; Bazgir,A. Synth Commun. 2009,39,1055-1064.

8. Guo,S.; Wang,S.; Li, J. Synth Commun. 2007, 37, 2111-2120.
9. Azarifar,D.; Maleki,B. Synth Commun. 2005, 35, 2581-2585.

10. Wang, S.; Ren, Z.; Cao, W.; Tong, W.; Synth Commun. 2001, 31, 673-677.

11. Bebernitz, G. R.; Argentieri, G.; Battle, B.; Brennan,C.; Balkan, B.; Burkey,B. F.; Eckhardt, M.; .Gao, J.; Kapa, P.; Strohschein,R. J.; Schuster,H. F.; Wilson, M.; Xu, D. D. J Med Chem. 2001, 44, 2601-2611.

12. Bekhit, A. A.; Fahmy H. T. Y.; Rostom, S. A. F.; Baraka, A.M. Eur J Med Chem. 2003, 38, 27-36.

13. Eid, A. I.; Kira, M. A.; Fahmy,H. H. J Pharm Belg. 1978, 33, 303-311.

14. Park, H.A.; Lee, K.; Park, S. J.; Ahn, B.; Lee, 
J.C.; Cho, H. Y.; Lee, K. I. Bioorg Med Chem Lett . 2005, 15, 3307-3312.

15. Parmar, S. S.; Pandey,B. R.; Dwivedic,C.; Harbison, R. D. J Pharm Sci. 1974, 63, 11521155.

16. Takabatake, E.; Kodama, R.; Tanaka,Y.; Dohmori, R.; Tachizawa,H.; Naito, T. Chem Pharm Bull. 1970, 18, 1900.

17. Boldi,A. M.; Johnson, C. R.; Eissa, H. O. Tetrahedron Lett. 1999, 40, 619.

18. Arroya,Y.; Rodriguez, J. F.; Santos, M.; Sanz M.; Tejedor, A.; Vaco, I.; GarciaRuano,J. L. Tetrahedron: Asymmetry. 2004, 15, 10591067.

19. Tanaka,S.; Seguchi, K.; Itoh, K. J Chem Soc, PerkinTrans. 1994, 1, 2335-2339.

20. Deghati,P.Y. F.; Wanner,M. J.; Koomen, G. J. TetrahedronLett. 1998, 39, 4561-4564.

21. Meehan, S.; Little, R. D. A. J Org Chem. 1997, 62, 3779-3781.

22. Menard,C.; Doris, E.; Mioskowski, C. Tetrahedron Lett. 2003, 44, 6591.

23. Nabid, M. R.; Tabatabaei Rezaei, S.J.; Ghahremanzadeh, R.; Bazgir, A.; Ultrason Sonochem. 2010,17, 159-161.

24. Azarifar, D.; Nejat-Yami, R.; Zolfigol, M. A. J. Hetero. Chem. 2013, 50, 1386-1390.

25. Adib, M.; Sayahi, M. H.; Mahmoodi, N.; Bijanzadeh, H. R.; Helv Chim Acta. 2006, 89, 1176-1180.
26. Ghahremanzadeh,R.; Shakibaei Imani, G.; Bazgir, A. Synlett. 2008,1129-1132.

27. Elinson, M.N.; Merkulova, V.M.; Ilovaiskya, A.I.; Barba, F.; Batanero, B. Electrochim. Acta. 2011, 56, 8219-8223.

28. Elinson, M M.N.; Dorofeev, A.S.S.; Feducovich, K.; Nasybullin, R.F.; Gorbunov,S.V.; Nikishin, G.I. Electrochem. Commun. 2006, 15671571 (b) Elinson, M.N.; Dorofeev, A.S.; Nasybullin,R.F.; Nikishin,G.I. Synthesis. 2008, 1933-1937.

29. Mousavi, M.R; Hazeri, N.; Maghsoodlou, M. T.; Salahi, S.; Habibi-Khorassani, S. M. Chin. Chem. Lett. 2013, 24, 411 -414.

30. Khandan-Barani, K.; Maghsoodlou, M. T.; Habibi-Khorassani, S. M.; Hazeri, N.; Sajadikhah, S. S. J Chem Res, 2011, 231238.

31. Khandan-Barani, K.; Maghsoodlou, M. T.; Habibi-Khorassani, S. M.; Hazeri, N.; Sajadikhah, S. S. Arkivoc, 2011, (xi), 22-28.

32. Khandan-Barani, K.; Maghsoodlou, M. T.; Hazeri, N.; Habibi-Khorassani, S. M. Iran. J. Org. Chem, 2012, 4, 901-904.

33. Elinson, M.N.; llovaisky,A.I.; Dorofeev, A.S.; Merkulova,V.M.; Stepanov,N.O.; Miloserdov, F.M.; Ogibin, Y.N.; Nikishin, G.I. Tetrahedron. 2007, 63, 10543-10548. 